\title{
Digital Direct Metallization (DDM) by a plasma coating process on polymeric components and its applications
}

\author{
Alexandru Sover*, Pilipp Popp, and Tamer Aydin \\ Ansbach University of Applied Sciences, Faculty of Engineering Sciences, Residenzstr. 8, Ansbach, \\ Germany
}

\begin{abstract}
Digital Direct Metallization (DDM) is an innovative process for the production of metallic layers on plastic substrates, e.g. to generate electrical circuit path. This paper describes the manufacturing process of Digital Direct Metallization (DDM) in the case of plasma based copper coating on polymeric components. The process-temperature has a significant impact on the metal deposition and can be controlled by the distance between the plasma head and plastic part or/and its cross-runs. The deposition of the copper powder on the polymeric surface was substantial improved by a temperature close to the melting point of the polymer.
\end{abstract}

\section{Introduction}

Digital Direct Metallization (DDM) is an innovative plasma process for the metal deposition on different substrates materials, planar or three-dimensional. The flexibility of this technology offers a great solution for the currently existing technologies of metal deposition on plastic materials and open new ways for different applications, i.e. design, electronic, sensors, telecommunication etc. [1-3].

The combination of plastic and metal on the surface of the parts can also improve the quality and functionality of the components concerning design, thermal and electrical properties, which are presented in different papers [1-4]. One of the new applications is the production of circuit layers, which can replace the conventional chemical metallization processes of the printed circuit board (PCB). The former is presented in papers [2-4] and applied by companies like Plasma Innovation.

Plasmadust ${ }^{\circledR}$ is a thermal spray technology using cold active plasma to melt a metal powder such as copper, which can be deposited directly on a surface [3]. This can be described as additive metallization technology.

Joerg Franke et. all present in different papers $[1,3]$ the importance of the particle size and the surface treatment with e.g. solder pastes and the interconnections between the substrate and the components in electronics [3]. Also the particle sizes of the metal powder of the process can highly influence the diffusion distances of the plasma jet [3].

\footnotetext{
*Corresponding author: a.sover@hs-ansbach.de
} 


\section{DDM Technology and used materials}

The main advantages of the Plasmadust ${ }^{\circledR}$ technologies are the variable process temperatures ranging between $90^{\circ} \mathrm{C}$ and $180^{\circ} \mathrm{C}$, the good adhesion of the metal layer, the high process speed of about $100 \mathrm{~mm} / \mathrm{s}$ and the fast growing metallic thickness with about $15 \mu \mathrm{m}$ thickness per cycle $[4,5]$. The settings of parameters like temperature, process speed or powder feed rate in the process have an important influence on the thickness of the metallic layer that can be achieved on thermoplastic or thermosets substrates [4-5]. Plastic specimens with or without surface treatment can be metallized with no or minimal thermal damage of the plastic surface, depending on these plasma process parameters [4].

The Plasmadust ${ }^{\circledR}$ technology can combine the surface treatment and metal deposition or can use plastic parts with an already mechanical prepared surface, e.g. one that is structured by laser (Laser-Direct-Structuring LDS). This provides the necessary surface structure for a better adhesion of the melted or partial melted metal particles [3]. The following picture presents the Plasmadust ${ }^{\circledR}$ technology by Plasma Innovations $\mathrm{GmbH}$.

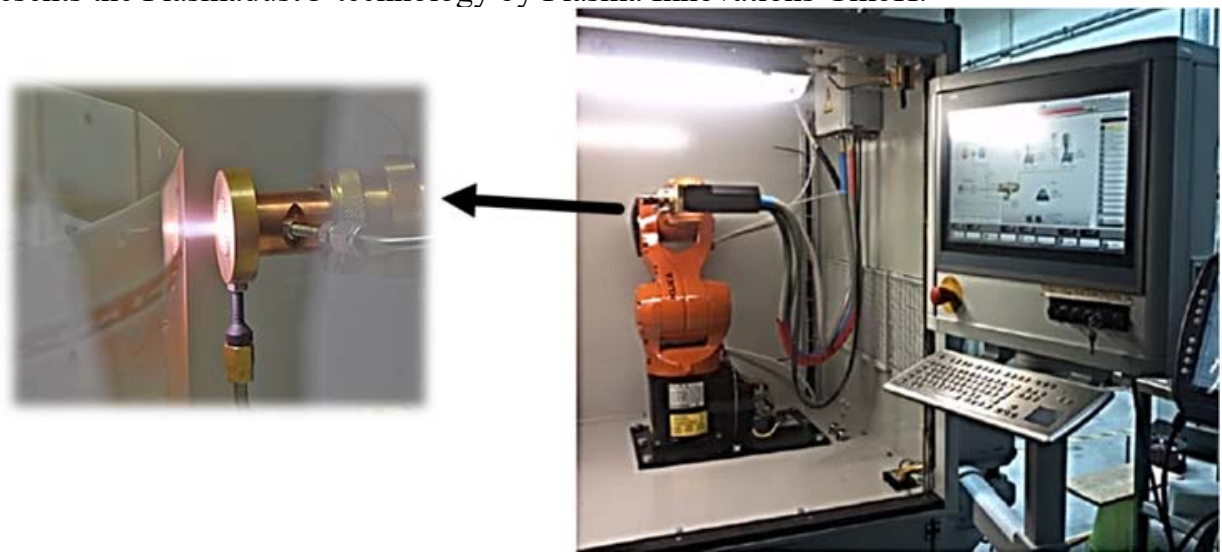

Fig. 1. Plasmadust ${ }^{\circledR}$ Equipment for Digital Direct Metallization (DDM).

The devices for active atmospheric plasma-metallization consists of a plasma head, installed on a robot arm, which can move in a small chamber in different directions, following the surface geometry of a product. For this machine argon gas is used as it can provide the desired plasma qualities but also due to its low costs and wide availability. The robot arm can be programmed to perform several move cycles to increase the thickness of the deposition and also for keeping a definite distance between the plasma head and surface, and the speed of movements can be adjusted. The following figure shows the plasma head with the powder feeders of the DDM equipment.

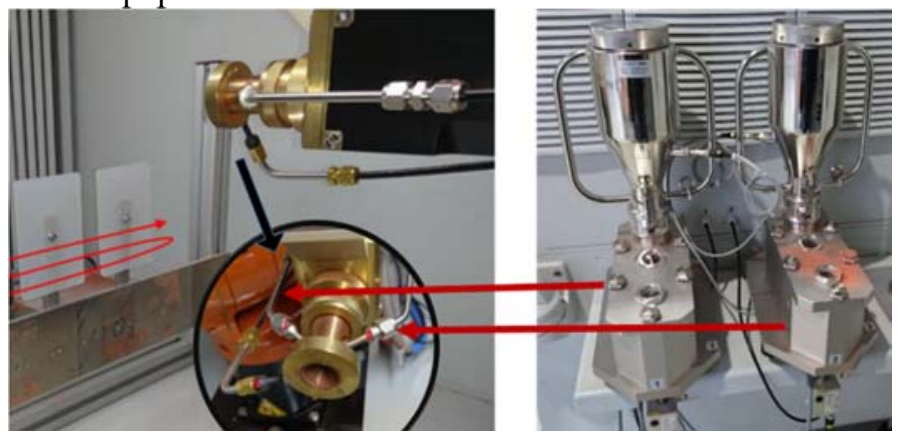

Fig. 2. Plasma head (left) for the DDM-Technique and the copper feeder (right). 
To avoid the thermal damage of the surface a certain distance between the plasma jet and surface is necessary, otherwise the polymeric surface can be deteriorated and the adhesion of the "printed" structures is negatively affected. The metallic powder used for the metallization is a special high tech copper powder produced by ECKART GmbH (member of ALTANA Group) with the trade name Plasmavario 1001 Copper [6]. This powder is a high purity copper $(99.9 \%)$ with very fine spherical particles (average particle size $10 \mu \mathrm{m}$ ) and narrow particle size distribution at a high electrical conductivity [6]. The copper powder is added to the plasma head where under high temperature it melts and from where it is projected with the argon gas onto the surface which should be metallized.

As polymer materials with different melting points were used in the experiments, the distance to the surface needed to be adjusted for each polymer to avoid the melting of the material but also depending of the surface treatment and copper dosage. At a distance between 10 and $30 \mathrm{~mm}$ the temperature on the specimen surface can vary between 80 to $120^{\circ} \mathrm{C}$ by per cycle (Fig. 2).

In the first test Polypropylen (PPH 9069) and Polyamide 6 (TECHNYL ${ }^{\circledR}$ C 206F) were used. The dimension of the prepared specimens was $100 \times 150 \times 4 \mathrm{~mm}$. The melting point of the both materials are $165^{\circ} \mathrm{C}$ for PP respectively $222^{\circ} \mathrm{C}$ for PA6.

The used parameters for the plasma deposition on the both polymer materials were:

- $\quad$ Distance plasma head to surface $(30,20$ and $10 \mathrm{~mm})$

- $\quad$ Moving speed of the plasma head $(0,3 \mathrm{~mm} / \mathrm{s})$

- $\quad$ Gas supply (25 1/min)

- $\quad$ carrier gas $(5 \mathrm{l} / \mathrm{min})$ for the feed rate copper

- feed rate 10,20 and $40 \%$

The plastic specimens were fixed on a device and half of the surface was pre-treated with plasma, immediately before the metal deposition, to improve the adhesion of the powder particles.

The third polymeric material tested was a Polyphenylen Sulfid (PPS) (Therma-Tech ${ }^{\mathrm{TM}}$ TT9200-5010 EC), a high temperature polymer with the melting point at $310^{\circ} \mathrm{C}$ and very good thermal management. The Therma-Tech ${ }^{\mathrm{TM}}$ compounds by the PolyOne Corporation improved thermal conductivity up to 100-times that of conventional plastics [7].

The PPS parts were coated with a thermoset varnish (PLASMACOAT PU41) which acted as an electric insulation layer between the metal layer and the circuit carrier. The electric insulation and non-stick varnish provided also the possibility to protect some areas from the metal deposition and allows the generation of the conductor lines for logic circuits. The coated layer can be structured or removed by a laser, to provide certain surface characteristics (roughness) for the metal deposition, Figure 7. The roughened substrate offers the necessary environment for a mechanical bond between the first melted or softened metal particles and the polymer. The coating thickness increased with each deposed metallic layer.

\section{Results}

The PP and PA plastic specimens were metallized at different parameters, as described above. The procedure is presented in the following figure in which two different prepared PA surfaces where metallized: a- no treatment, b- with plasma treatment. 

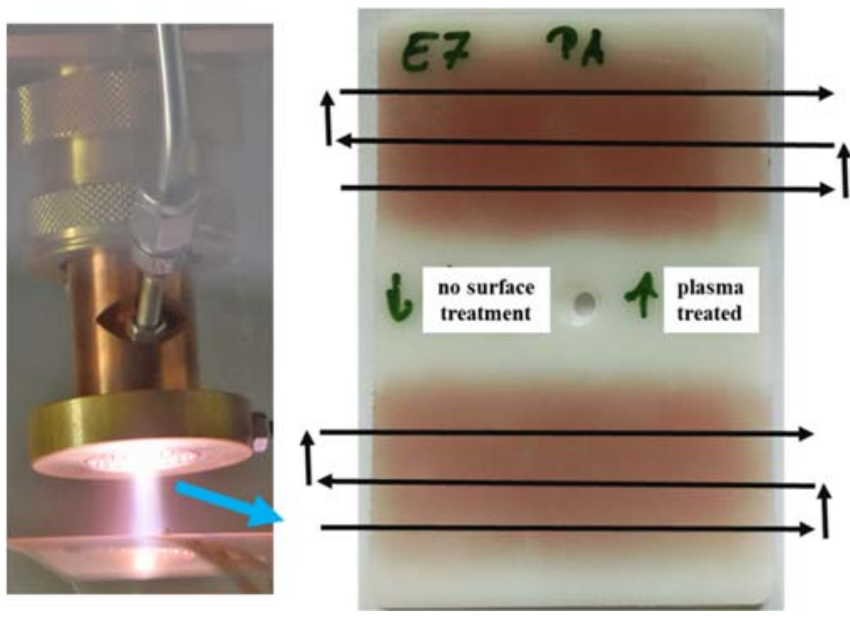

Fig. 3. PA specimen with (top) and without plasma surface treatment (down) at plasma metallization with the path of the plasma head.

The arrows show the path of the plasma head for the metal deposition. The temperature on the specimen surface showed values between $90^{\circ} \mathrm{C}$ (first run) and $124^{\circ} \mathrm{C}$ (third run) at a distance of $30 \mathrm{~mm}$ [6]. The deposition of the copper on the PP and PA specimens was hardly influenced by the distance between the plasma head and the surface.

At a distance of $30 \mathrm{~mm}$ only a thin cooper layer with the dimension of ca. $10 \mu \mathrm{m}$ was observed, which is also the average copper particle size. Also, the particles kept their spherical form, which indicates that they were not completely melted before the deposition. The adhesion of the metallic layer was very poor; it could be partially removed with a finger. When the distance between the plasma head and specimen surface from 30 to $10 \mathrm{~mm}$ was decreased an improvement of the deposition was observed for both samples (PP and PA).The PP sample showed even better deposition compared to the PA sample (Fig. 5).

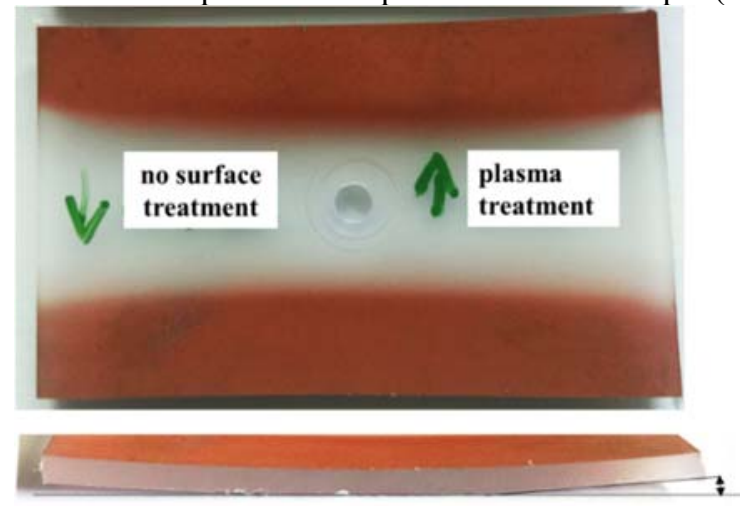

Fig. 4. Local copper metallized PP samples with and without surface treatment at $10 \mathrm{~mm}$ distance between plasma head and surface [5].

The adhesion of the copper particles on the PP surface was much better compared to the adhesion to the PA samples. A reason for this effect can be the lower melting point of the PP material (ca. $165^{\circ} \mathrm{C}$ ), which facilitates a local melting of the PP polymer and the copper particles can be better enclosed in the surface. An evidence of the high temperature exposure of the surface is the warpage of the plate specimens, with was more than $1 \mathrm{~mm}$, Figure 5 above. Also, the microscopically comparison of the copper layer on the PP surface at 30 and 
$10 \mathrm{~mm}$ plasma head distances shows different types of adhesion of the copper particles on the material surface.
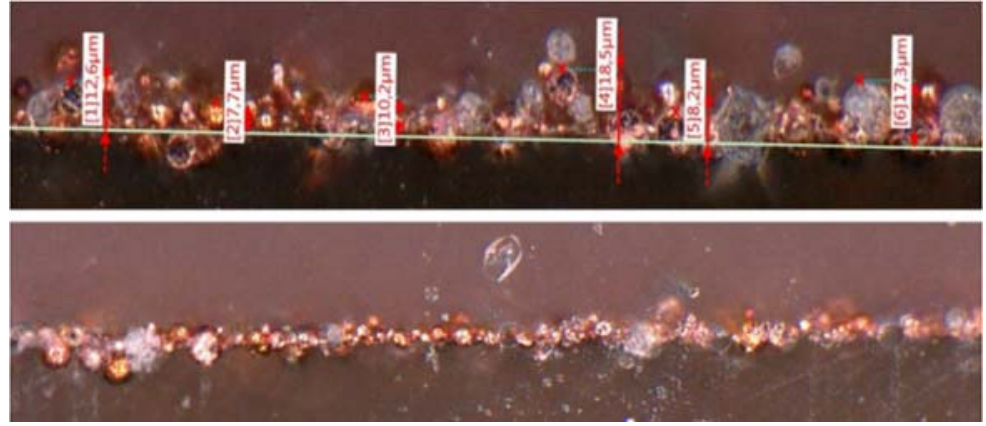

Fig. 5. Copper Layer on PP specimen by 30 and $10 \mathrm{~mm}$ distance of the plasma head to the surface [5].

In the upper image of Figure 6 one can see that at higher temperatures on the polymer surfaces the copper particles are enclosed in the plastic material. This increases the adhesion of the first metallic layer, but it can also change the dimensions of the plastic parts Figure 5. When the powder feed rate was increased up to $40 \%$ the metallic layer became thicker between 20 and $40 \mu \mathrm{m}$.

The next figure shows the circuit carrier after the copper depositing processes. The PPS material was coated with an anti-adherent varnish and after drying the circuit path was structured / removed by IR-laser.
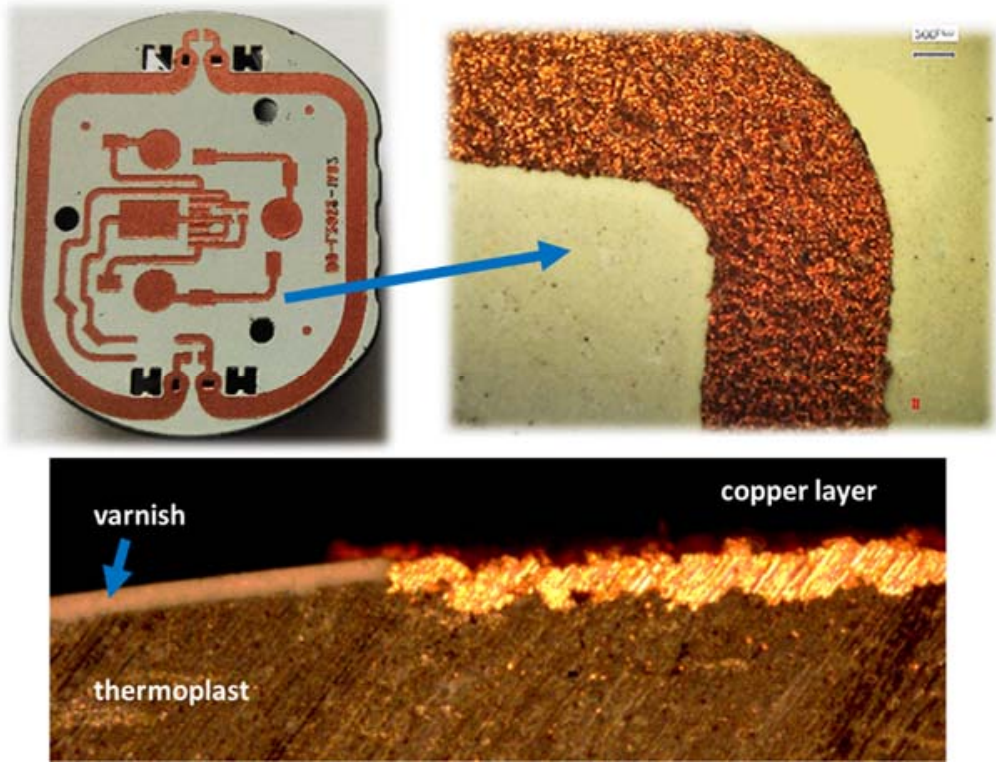

Fig. 6. LED plastic carrier produced with MID technique by DDM metallization [8].

Figure 7 shows a cross section through the part, where the varnish and copper layer on the PPS polymer can be seen. The copper particles are adhering on the surface structure without thermal damage of the substrate and form a continuous coating. The particles are melted by plasma and accelerated to the surface by the gas jet where they solidify and build a mechanically bond, firstly with the roughened surface and then with each other as the coating thickness is increased. At a higher optical magnification, the spherical geometry of the particles can be perceived, which shows that the particles are not completely melted. The 
layer is electrically conductive and the high thermal resistance of the plastic materials allows a higher process temperature which results in a better adhesion. With these types of polymers, the metallization of the surface can be done more easily without other warpage effects on the components.

\section{Conclusion}

The deposition of copper on three different polymeric substrates (PP, PA, and PPS) was tested under different conditions. Without any surface structuring the adhesion of the copper substrate depends mostly on the thermal proprieties of the polymeric material. A higher temperature on the polymer surface, close to his melting point (e.g. $165^{\circ} \mathrm{C}$ for PP) leads to a better deposition of the copper powder on the surface. At higher temperatures the copper particles can easily be integrated in the melted polymer film, but the geometry of the plastic specimen can suffer a highly (more millimeters) warpage. On the other hand, a structured surface can provide a mechanical bond for the melted or partially melted copper particles. The plasma pre-treatment of the surfaces did not significantly improve the adhesion of the copper layer on the examined PP and PA polymers.

A special gratitude I give to the students of the HS Ansbach who contributed in different steps with their work to this research project. Special thanks for her support go to Ms Antje Sover.

\section{References}

1. J. Franke, Materials, Manufacturing, Assembly and Applications for Injection Molded Circuit Carriers, Carl Hanser Verlag (2014)

2. M. Mueller, J. Franke, Spring Seminar on Electronics Technology (2016)

3. J. Franke, A. Syed-Khajaa, R. Schramm, R. Ochs, Packaging through Additive Plasma Technology, Procedia CIRP, 37 (2015)

4. R. Schramm, J. Franke, Electronics Packaging Technology Conference (2013)

5. P. Popp, T. Aydin, Plasma Oberflächenbehandlung, Projektarbeit an der HS Ansbach, (2017)

6. U.-A. Hirth, High Tech Copper Powder, for Digital Direct Metallization, Technical Seminar at kunststoffcampus Bayern (2016)

7. J. Kuntermann, Thermally conductive plastics: Challenges, experience and the road ahead, Technical Seminar at kunststoffcampus Bayern (2016)

8. M. Horn, H. Hanson, Voruntersuchung und Testverfahren zur Beurteilung der Eigenschaften von plasmabeschichteten MID-Bauteilen, Projektarbeit HS-Ansbach (2016) 